\title{
Religion und Populärkultur im 21. Jahrhundert - Differenzierungen und Entgrenzungen
}

\author{
Meike Haken · Kornelia Sammet
}

Angenommen: 19. Oktober 2021 / Online publiziert: 3. Dezember 2021

(C) Der/die Autor(en) 2021

Die Frage nach Rolle und Bedeutung populärer Kultur für Phänomene im Bereich der Religion ist seit den 1990er-Jahren insbesondere im angelsächsischen Raum Gegenstand sowohl einzelner sozial-, kultur- und geisteswissenschaftlicher Studien (z. B. McDannell 1995; Lynch 2007; Possamai 2005; Partridge 2009) als auch ganzer Kompendien (z. B. Fechtner 2005; Schlehe und Sandkühler 2014; Lyden und Mazur 2015). Was dabei aber dieses „Populärkulturelle“ ist, bleibt zumeist unklar, und es herrscht - verstärkt durch den Einfluss angelsächsischer Forschung im Zeitalter von Globalisierungsprozessen - kaum Einigkeit darüber. Im deutschsprachigen Raum können seit Ende des letzten Jahrtausends grob zwei Perspektiven auf das Verhältnis von Religion und Kultur im Allgemeinen sowie Religion und populärer Kultur im Besonderen ausgemacht werden. Auf der einen Seite wird - in Anschluss an Max Webers Unterscheidung von Wertsphären ([1920] 1988, S. 536-573) mit ihren jeweiligen ,,innere(n) Eigengesetzlichkeiten“ (541) und Logiken (Tyrell 2017; Sammet 2020, S. 161ff.) sowie an Luhmanns Theorie funktionaler Differenzierung Religion in Hinblick auf ihre Funktion und ihr Bezugsproblem sowie den damit verbundenen Semantiken und Codes von anderen gesellschaftlichen Teilbereichen unterschieden (Luhmann 1987, 1998; Pollack 1995; Krech 2019). Wechselseitige Anleihen von Religion und Populärkultur sind dementsprechend als Grenzarbeiten oder (De-)Sakralisierungsprozesse zu verstehen (Krech 2019). Eßbachs (2014) kompetetive Differenzierung arbeitet in historischer Perspektive heraus, wie neue Formen von Religion, die aufgrund der zunehmenden Bedeutung des Populären entstehen und die Grenzen zu anderen Gesellschaftsbereichen verwischen, mit bereits bestehenden Formen in Konkurrenz treten.

\footnotetext{
Meike Haken $(\triangle)$

Technische Universität Berlin, Sekretariat FH 9-1, Fraunhoferstr. 33-36, 10587 Berlin, Deutschland

E-Mail: meike.hellmuth@freenet.de

Kornelia Sammet

Deutsches Jugendinstitut e.V, Franckeplatz 1, Haus 12/13, 06110 Halle, Deutschland
} 
Auf der anderen Seite wird eine zunehmende Entgrenzung kulturell geformter Wissensformationen verschiedener gesellschaftlicher Teilbereiche beobachtet. Dementsprechend nimmt die These der Entgrenzung - wie auch differenzierungstheoretische Zugänge - kulturelle Formen und Inhalte von Kommunikation generell und damit auch der religiösen Kommunikation in den Blick. Der Begriff der Popularisierung bezieht sich nun explizit auf die Kultur. Hubert Knoblauch hat im Jahr 2009 in Populäre Religion. Auf dem Weg in eine spirituelle Gesellschaft seine vielfältigen empirischen Arbeiten zu einem religionssoziologischen Konzept weiterentwickelt, mit dem sich Entgrenzungen zwischen Religion und populärer Kultur verstehen lassen. Vor diesem Hintergrund kamen zunehmend Fragen in Hinblick auf die Angemessenheit und Reichweite von Zeitdiagnosen wie der Profanisierung der Religion, der Desakralisierung oder Resakralisierung auf. Insgesamt wurde die Populäre Religion als Werkzeug rezipiert, und zwar transdisziplinär sowohl innerhalb der Religionsethnographie (z.B. Gründer 2010; Rettig 2017) als auch der Religionssoziologie (z. B. Schnettler 2014; Schnettler und Heid 2014; Haken 2017, 2019, 2020) und der Kulturwissenschaft (z. B. Zink 2014, 2019; Neufend 2019).

Die im November 2019 an der Technischen Universität Berlin von Robert Schäfer, Kornelia Sammet und Hubert Knoblauch veranstaltete Jahrestagung der DGSSektion Religionssoziologie zum Thema „Religion und Populäre Kultur“ machte einerseits wiederum die Diversität des Begriffs des Populären bzw. der populären Kultur deutlich, andererseits jedoch auch, dass sich in den letzten Jahren die Forschung zum Zusammenhang von Religion und populärer Kultur auf eine neue Ebene bewegt hat. Erschien es zeitweise als unhinterfragt, dass es Phänomene im Bereich des Religiösen und Spirituellen gibt, bei denen sich deutliche Einflüsse aus anderen kulturellen Bereichen erkennen lassen, so wird gegenwärtig auch die Relation zwischen populärkulturellen religiösen Formen einerseits und als traditionell religiös deutbaren, oftmals kirchlicher Religion entstammenden Formen und Phänomenen andererseits vermehrt in empirischen Studien beleuchtet. Zugleich werden in der Forschung neue theoretische Konzepte und methodische Zugänge aufgegriffen, die religionssoziologische Debatten bereichern können. Dadurch rückt auch das Verhältnis kultur- und differenzierungstheoretischer Perspektiven auf Religion und populäre Kultur auf neue Weise zurück ins Zentrum der Diskussion.

Dieses Sonderheft umfasst eine Reihe von empirisch fundierten konzeptionellen Arbeiten, die sich entweder das eine oder das andere zur Aufgabe machen. Dabei werden grundlegende Fragen zur Definition von populärer Kultur und ihrem Verhältnis zur Religion vor dem Hintergrund globaler gesamtgesellschaftlicher Transformationsprozesse gestellt. Doch wird ebenfalls eruiert, welche Bedeutung traditionellen, insbesondere kirchlich-religiösen Ritualen, Praktiken und Institutionen innerhalb gegenwärtiger Erscheinungsformen von Religion zukommt und wie sie insbesondere über digitale Mediatisierung transformiert werden. Die Beiträge zeigen, dass der Einfluss des Populärkulturellen nicht nur zu einer Entgrenzung im Bereich der Religion führen kann, sondern - über die verschiedenen Sozialformen des Religiösen hinweg beobachtbar - Dynamiken der Ausdifferenzierung und eine stärkere Markierung von Religion nicht ausschließt. Diese Formen der Betonung der Spezifik des Religiösen zeigen sich in den Studien der Autorinnen und Autoren an räumlichen, medialen und kommunikativen Inszenierungen von Einzelakteuren 
und/oder Gemeinschaften. Die Markierungen stellen dabei eine Antwort auf die beobachtbaren Entgrenzungsprozesse der letzten Dekaden dar (vgl. Haken 2020) und verlangen in der Forschung den konzeptionellen Einbezug auch vermeintlich gegenläufiger Transformationen gesellschaftlicher Kommunikationsprozesse. Alle Beiträge reichen über einen illustrativen deskriptiven Einblick hinaus, vielmehr eröffnen sie durch ihre Theoriearbeit neue Anschlusspunkte für religionssoziologische Forschung.

Die Beiträge des Sonderheftes lassen, unabhängig vom fokussierten Gegenstand, verschiedene Kategorien deutlich hervortreten: Inszenierungen von Einzelakteuren und Gemeinschaften werfen Fragen nach Authentizität und Subjektivierungspraktiken auf. Ausschlaggebend für derartige Prozesse sind - wie Knoblauch in seinem Beitrag argumentiert - Transformationen des Medialen, die sich im Zeitalter der Digitalisierung als digitale Mediatisierung zeigen. Raum und Architektur sind Aspekte, die bislang - wenn überhaupt - nur marginal oder vor allem bezogen auf kirchlich-materielle Manifestationen in der Religionssoziologie Beachtung fanden (Karstein und Schmidt-Lux 2017), in diesem Sonderheft aber in unterschiedlichster Weise bearbeitet werden. Die Bedeutung des Räumlichen für Erscheinungsformen von Religion und Spiritualität im 21. Jahrhundert wird u. a. an religiösen Events, aber auch der zunehmenden Touristifizierung des Religiösen deutlich. All diese Beobachtungen führen zur - wenn auch unterschiedlich ausgestalteten - Diagnose einer Veränderung der religiösen Kommunikationskultur. Es handelt sich bei den Beiträgen dieses Sonderheftes um empirische Arbeiten, die sich in unterschiedlicher Weise dem erhobenen (Bild-)Material nähern. Insofern weist das Sonderheft nicht nur eine theoretische Vielfalt zum Thema Populärkultur und Religion auf, sondern ist auch, was die methodischen Herangehensweisen angeht, breit aufgestellt.

Im ersten Beitrag befasst sich Carsten Heinze mit dem Zusammenhang von Religion und Metaphysik im Werk Siegfried Kracauers. Medien - hier: Kino, Film und Fotografie - werden als Ausdruck populärer Kultur in ihrer Bedeutung für die Moderne betrachtet. Religion kommt insbesondere in einer funktionalen Sichtweise ins Spiel, denn im Kern fragt der Autor nach der Bearbeitung und Substitution des Erlösungsmoments durch den Film als funktionales Äquivalent. Von den genannten Kernkategorien dieses Sonderheftes, die sich als roter Faden durch die Beiträge ziehen, kommen Kommunikation und Medien insbesondere über den inhaltlichen Fokus, aber auch den Analysegegenstand - Krakauers Werke - zum Tragen.

Explizit wird das Verhältnis von Religion und Populärkultur von Luis Bastidas, Tom Kaden und Bernt Schnettler aufgegriffen. Basierend auf langfristigen ethnographischen Erhebungen wird der kolumbianische „Kult der Seelen im Fegefeuer“ in Puerto Berrío und seine Beziehung zur katholischen Kirche untersucht. Dabei wird anhand dreier Merkmale dieses Kults ein Vergleich mit weiteren Formen des lateinamerikanischen populären Katholizismus vorgenommen. Die Form des Populären, die Religion hier annimmt, kann theoretisch zunächst mit Luckmanns Unsichtbare( $r$ ) Religion (1996 [1991]) und Knoblauchs Populäre(r) Religion (2009) begriffen werden. Die Analyse arbeitet jedoch sehr viel stärker die damit verfolgte Abgrenzung von traditionellen institutionalisierten Formen des Katholizismus heraus. Der „Kult der Seelen“ steht exemplarisch für eine zweideutige Beziehung zur Katholischen Kirche, die zwischen Angleichung und Opposition wechselt. 
Eine Art Wechselspiel zwischen individueller Aneignung bzw. Inszenierung einerseits und institutioneller Einbindung von Religion andererseits findet sich auch in Patrick Heisers Beitrag zum Thema Fasten. Aus der Perspektive des ,lived religion“Ansatzes wird auf Basis quantitativer Daten zum Fasten in Deutschland herausgestellt, dass das Fasten heutzutage zwar eine grenzüberschreitende Praktik ist, der Zeitraum des Fastens durch die Bindung an konfessionelle Fastenzeiten jedoch eine explizit gelebte religiöse Praxis daraus macht.

Um eine spezifische Praxis geht es auch im Beitrag von Kornelia Sammet und Uta Karstein, die mit dem Thema Touristifizierung von Religion ein noch wenig beforschtes Terrain in der Religionssoziologie betreten. Mit der Untersuchung religiös markierter touristischer Räume greifen die Autorinnen ein zweites relativ junges Thema in der Religionssoziologie auf. Anhand des Wanderns als einer spezifischen Form der touristischen Bewegung in landschaftlich reizvoller Umgebung wird aus differenzierungstheoretischer Sicht die Frage danach gestellt, wie durch den Tourismus auf Religiöses Bezug genommen und Religion touristisch recodiert wird. Analysegegenstand sind verschiedene Objektivationen in Gestalt touristischer Wegbeschreibungen, aus denen kommunikative Muster touristischer Bezugnahmen auf Religiöses, aber auch religiöse Recodierungen des Touristischen sequenzanalytisch rekonstruiert werden.

Raum wird als Analysekategorie von Marian Burchardt und Johanna Häring anhand des Verhältnisses von Macht, Religion und Stadtraum aufgegriffen. Der Beitrag basiert auf Gruppendiskussionen und Interviews mit unterschiedlichen Akteursgruppen, die die Wahrnehmung interreligiöser Beziehungen und die Positionierung zu interreligiösen Dialogen zum Gegenstand hatten. Als Analysegegenstand dient das Berliner „House of One“, das als multireligiöse Architektur in den letzten Jahren Aufmerksamkeit erlangt hat. Kernargument ist dabei, dass innerhalb der analysierten Diskurse kulturelle Hierarchien zu Tage treten, die bislang im Rahmen des DialogParadigmas wenig Betrachtung fanden.

Der Beitrag von Silke Steets basiert auf Daten, die im Rahmen einer ethnographischen Studie im texanischen Waco erhoben wurden. Er befasst sich mit der Transformation von Kommunikationsstrategien unter Hinzunahme einer raumsoziologischen Perspektive. Damit wird auch hier an jüngste religionssoziologische Diskurse angeknüpft. Anhand einer TV-Heimwerker-Serie, in der für zuvor ausgewählte Menschen renovierungsbedürftige Häuser verschönert werden, arbeitet die Autorin ein Narrativ heraus, das über spezifische Erzählstrukturen religiöse Züge aufweist. So weist z.B. der „Demolition Day“, bei dem es um Abrissarbeiten in den Häusern geht, strukturelle Ähnlichkeiten mit einem Reinigungsritual auf. Der Beitrag zeigt, welchen Einfluss räumliche Strukturen, auch in ihrer historischen Entwicklung, über populärkulturelle Kommunikationsformen auf Religion haben.

Insa Pruisken und Josefa Loebell vergleichen - ausgehend von der These der Refusion differenzierter Elemente kollektiver Rituale innerhalb einer Inszenierung in ihrer qualitativen Untersuchung unterschiedlicher evangelikaler Sozialformen von Religion in den USA drei kollektive Rituale miteinander. Hier stehen insbesondere die Kategorien Mediatisierung und Digitalisierung als Transformationsmotoren des Religiösen im Zentrum der Betrachtung einer traditionellen Gemeinde, einer Megakirche und einer Vloggerin. Ebenso wie in Steets' Beitrag zielt die Analyse 
auf Kommunikationsformen und die Rolle der Medien bei dieser Transformation. Besondere Aufmerksamkeit kommt in diesem Zusammenhang einem in der Religionssoziologie etwas vernachlässigten Aspekt zu. Authentizität wird hier, ähnlich wie es in der Theologie vertreten wird, als Kernelement dessen, was als religiös bezeichnet werden kann, betrachtet.

Auch im Beitrag von Jonathan Kühn und Henrik Simojoki begegnet uns das Thema Authentizität in der Analyse einer spezifischen medialen Form des Religiösen. Wie auch bei Pruiskens und Loebells drittem Fall geht es um die Inszenierung einer populären Sinn-Fluencerin, hier im Rahmen des Kanals „Jana glaubt“, der allerdings nicht von evangelikaler, sondern von amtskirchlicher Seite aus initiiert wurde. Theoretisch wird Knoblauchs Konzept der Populären Religion über einen interdisziplinären Mediatisierungsansatz mit dem praktisch-theologischen Leitbegriff der Kommunikation des Evangeliums verknüpft. Analysiert wird die dargestellte spezifische Kommunikationsform mit der wissenssoziologischen Videohermeneutik.

Das Thema digitale Medien steht auch im folgenden Beitrag von Adrian Totaro im Zentrum der empirischen Analyse, die theoretisch ebenfalls an das Konzept der Populären Religion anknüpft. Anhand des Videos einer freikirchlichen Gemeinde werden mittels wissenssoziologischer Videohermeneutik drei Ebenen des Videos untersucht: seine mediale Gestalt, die darin körperlich und sprachlich vollzogene Handlung und ihr räumliches Setting. Auf der Basis der Dateninterpretation wird ein spezifischer Inszenierungstyp herausgearbeitet. Die Verquickung von Religion und Säkularität wird dabei über das Wechselspiel von traditioneller massenmedialer Kommunikation und neuen kreativen Formen deutlich. Wie im Beitrag von Steets wird auch in Totaros Studie die Rolle des Räumlichen für Religion hervorgehoben.

Mediatisierung, Authentizität und kommunikative Strategien der Inszenierung sind auch die Kernkategorien des Beitrags von Gabriel Malli. Theoretisch wird hier allerdings anders als in den bisherigen Beiträgen aus einer poststrukturalistischen Perspektive die Inszenierung von Weiblichkeit durch muslimische Vloggerinnen innerhalb von YouTube-Kanälen betrachtet. Im Zentrum des Interesses stehen die im Diskurs generierten Subjektpositionen muslimischer Weiblichkeit.

Das Thema Weiblichkeit und Mediatisierung wird auch von Maren Freudenberg und Dunja Sharbat Dar in ihrem Beitrag aufgenommen. Anhand der OnlinePlattform „Ladies Lounge“ einer evangelikalen Freikirche im deutschsprachigen Raum rekonstruieren sie, wie konservative christliche Gemeinschaften populärkulturelle Medienformate nutzen, um traditionelle Geschlechterrollen zeitgenössisch zu reinszenieren. Der empirische Beitrag nutzt zur Analyse des Bildmaterials die objektive Hermeneutik, theoretisch verfolgt er eine postfeministische Herangehensweise.

Alle Beiträge dieses Sonderheftes unternehmen eine sowohl empirische als auch theoretische Hinwendung zu verschiedenen Aspekten gegenwärtiger Erscheinungsformen von Religion. In ihren Analysen spielen die Bedeutung von Authentizität, die Dimension des Räumlichen sowie Dynamiken der Eventisierung, Mediatisierung und Digitalisierung eine große Rolle. Dabei zeigen sich diese Kategorien in der Beobachtung religiöser Kommunikation. Knoblauchs abschließender Beitrag setzt sich nicht nur kritisch mit dem Begriff der populären Kultur auseinander, sondern auch mit seinem eigenen Konzept der Populären Religion. In seiner Argumentation konzentriert er sich nicht nur auf die digitale Mediatisierung und stellt damit die 
Digitalisierung als besondere Form der Mediatisierung heraus. Mit dem Begriff der „Refiguration“, der zunächst auf gesamtgesellschaftliche und räumliche Veränderungen hinweist, bringt er die Religion mit diesen Veränderungsprozessen zusammen. Knoblauchs Beitrag nimmt damit theoretisch auf, was sich empirisch unabhängig voneinander in den Beiträgen abzeichnet.

Danken möchten wir zunächst den Autorinnen und Autoren, die viel Zeit und Energie in die Ausarbeitung, Überarbeitung und Ausweitung der z. T. bei der Sektionstagung präsentierten Überlegungen gesteckt haben, vor allem jedoch den anonymen Gutachterinnen und Gutachtern, die die Beiträge mit ihren Hinweisen schnell und gewissenhaft bereichert haben. Besonderer Dank gilt auch Hubert Knoblauch und Robert Schäfer, ohne deren Vorarbeit zur Tagung die Idee zu diesem Sonderheft nicht aufgekommen wäre. Zu guter Letzt sei noch Heiko Beyer, dem Redakteur der Zeitschrift für Religion, Gesellschaft und Politik, für die reibungslose und angenehme Zusammenarbeit gedankt.

Funding Open Access funding enabled and organized by Projekt DEAL.

Open Access Dieser Artikel wird unter der Creative Commons Namensnennung 4.0 International Lizenz veröffentlicht, welche die Nutzung, Vervielfältigung, Bearbeitung, Verbreitung und Wiedergabe in jeglichem Medium und Format erlaubt, sofern Sie den/die ursprünglichen Autor(en) und die Quelle ordnungsgemäß nennen, einen Link zur Creative Commons Lizenz beifügen und angeben, ob Änderungen vorgenommen wurden.

Die in diesem Artikel enthaltenen Bilder und sonstiges Drittmaterial unterliegen ebenfalls der genannten Creative Commons Lizenz, sofern sich aus der Abbildungslegende nichts anderes ergibt. Sofern das betreffende Material nicht unter der genannten Creative Commons Lizenz steht und die betreffende Handlung nicht nach gesetzlichen Vorschriften erlaubt ist, ist für die oben aufgeführten Weiterverwendungen des Materials die Einwilligung des jeweiligen Rechteinhabers einzuholen.

Weitere Details zur Lizenz entnehmen Sie bitte der Lizenzinformation auf http://creativecommons.org/ licenses/by/4.0/deed.de.

\section{Literatur}

Eßbach, Wolfgang. 2014. Religionssoziologie 1. Paderborn: Fink.

Fechtner, Kristian. 2005. Handbuch Religion und Populäre Kultur. Stuttgart: Kohlhammer.

Gründer, René. 2010. Blótgemeinschaften. Eine ReligionsEthnographie des, germanischen Neuheidentums'. Würzburg: Ergon.

Haken, Meike. 2017. Religiöse Kommunikation in der Konstruktion christlicher Großveranstaltungen. In Der kommunikative Konstruktivismus bei der Arbeit, Hrsg. Jo Reichertz, René Tuma, 160-187. Weinheim, Basel: Beltz Juventa.

Haken, Meike. 2019. Religious emotions in Christian events. In Affect and emotion in multi-religious secular societies, Hrsg. Christian von Scheve, Anna Lea Berg, Meike Haken, und Nur Yasemin Ural, 114-132. London: Routledge.

Haken, Meike. 2020. Die kommunikative Konstruktion religiöser Events und ihre affektive Ordnung. In Die Refiguration der Religion. Perspektiven der Religionssoziologie und Religionswissenschaft, Hrsg. Hubert Knoblauch. Weinheim: Beltz Juventa.

Karstein, Uta, und Thomas Schmidt-Lux (Hrsg.). 2017. Architekturen und Artefakte. Zur Materialität des Religiösen. Wiesbaden: Springer VS.

Knoblauch, Hubert. 2009. Populäre Religion. Auf dem Weg in eine spirituelle Gesellschaft. Frankfurt a.M.: Campus. 
Krech, Volkhard. 2019. Operative Durchlässigkeit oder Schließung der Religion? Eine differenzierungstheoretische Lesart der Religionssoziologie von Wolfgang Eßbach. Zeitschrift für Religion, Gesellschaft und Politik 3(1):103-130.

Luckmann, Thomas. 1996. Die Unsichtbare Religion. Frankfurt a.M.: Suhrkamp. [1991].

Luhmann, Niklas. 1987. Die Unterscheidung Gottes. In Soziologische Aufklärung 4 Beiträge zur funktionalen Differenzierung der Gesellschaft., Hrsg. Niklas Luhmann, 236-253. Opladen: Westdeutscher Verlag.

Luhmann, Niklas. 1998. Religion als Kommunikation. In Religion als Kommunikation, Hrsg. Hartmann Tyrell, Volkhard Krech, und Hubert Knoblauch, 135-145. Würzburg: Ergon.

Lyden, John C., und Eric Michael Mazur (Hrsg.). 2015. The Routledge companion to religion and popular culture. London: Routledge.

Lynch, Gordon. 2007. Between sacred and profane: researching religion and popular culture. London: Tauris.

McDannell, Colleen. 1995. Material christianity: religion and popular culture in America. New Haven: Yale Univ. Press.

Neufend, Maike. 2019. The practice of vision: Sufi aesthetics in everyday life. In Affect and emotion in multi-religious secular societies, Hrsg. Christian von Scheve, Anna Lea Berg, Meike Haken, und Nur Yasemin Ural, 96-114. London: Routledge.

Partridge, Christopher. 2009. Religion and popular culture. In Religions in the modern world, Hrsg. Linda Woodhead, Hiroko Kawanami, und Christopher Partridge, 489-522. London: Routledge.

Pollack, Detlef. 1995. Was ist Religion? Probleme der Definition. Zeitschrift für Religionswissenschaft 3:163-190.

Possamai, Adam. 2005. Religion and popular culture: a hyper-real testament. Bruxelles: Peter Lang.

Rettig, Hanna. 2017. Making Missionaries - Junge Evangelikale und ihre Mission. Ethnographie einer Jugendorganisation auf Reisen. Bielefeld: transcript.

Sammet, Kornelia. 2020. Religion und Geschlechterordnungen in der Religionssoziologie um 1900. In Religionssoziologie um 1900. Eine Fortführung, Hrsg. Volkhard Krech, Hartmann Tyrell, 125-170. Baden-Baden: Ergon.

Schlehe, Judith, und Evamaria Sandkühler. 2014. Religion, tradition and the popular: Transcultural views from Asia and Europe. Bielefeld: transcript.

Schnettler, Bernt. 2014. Transzendenzerfahrung und populäre Religion. In Zwischen Säkularisierung und religiöser Vitalisierung: Religiosität in Deutschland und Polen im Vergleich, Hrsg. Michael Hainz, Gert Pickel, Detlef Pollack, Maria Libiszowska-Żółtkowska, und Elżbieta Firlit, 271-277. Wiesbaden: Springer VS.

Schnettler, Bernt, und Michaela Heid. 2014. Reisen mit spirituellen Sinnangeboten: Explorationen im Feld moderner Pilgerformen. In Pilgern gestern und heute: Soziologische Beiträge zur religiösen Praxis auf dem Jakobsweg, Hrsg. Patrick Heiser, Christian Kurrat, 221-243. Berlin, Münster: LIT.

Tyrell, Hartmann. 2017. Die Religion der Zwischenbetrachtung. Max Webers ,spezifisch religiöse Liebesgesinnung'. In Religion soziologisch denken. Reflexionen auf aktuelle Entwicklungen in Theorie und Empirie, Hrsg. Heidemarie Winkel, Kornelia Sammet, 347-384. Wiesbaden: Springer VS.

Weber, Max. 1988. Gesammelte Aufsätze zur Religionssoziologie I. Tübingen: Mohr. [1920].

Zink, Veronika. 2014. Von der Verehrung. Frankfurt a.M.: Campus.

Zink, Veronika. 2019. On conversion: affecting secular bodies. In Inside out: affect and emotion in multi-religious secular societies, Hrsg. Christian von Scheve, Anna Lea Berg, Meike Haken, und Nur Yasemin Ural, 132-151. London: Routledge. 